\title{
ASOCIACIÓN ENTRE ROTAVIRUS Y LA PRESENCIA DE DIARREA EN LECHONES DE GRANJAS TECNIFICADAS
}

\author{
Association between Rotavirus and the Presence of Diarrhea in Piglets \\ From InTEnsive Pig F ARMS
}

\author{
Miguel Rojas M. ${ }^{1}$, Alberto Manchego S. ${ }^{1,2}$, Hermelinda Rivera G. ${ }^{1}$, Néstor Falcón \\ P. ${ }^{3}$, Mercy Ramírez V. ${ }^{1}$, Nieves Sandoval Ch. ${ }^{4}$
}

\section{Resumen}

\begin{abstract}
El objetivo del estudio fue cuantificar la frecuencia del rotavirus y su asociación con la presentación de diarrea en lechones de dos granjas tecnificadas en la zona de Lima. El rotavirus fue identificado mediante la detección del genoma viral por electroforesis en gel de poliacrilamida (PAGE) en muestras de heces diarreicas $(n=69)$ y no diarreicas $(n=73)$ de lechones de 1 a 4 semanas de edad. Se empleó el diseño epidemiológico caso-control no pareado para establecer la asociación entre presentación de diarrea y presencia de rotavirus en heces, a través de una prueba de regresión logística múltiple (tipo de heces, procedencia, edad). La frecuencia de rotavirus porcino grupo A en heces diarreicas de la granja 1 fue de $41.4 \%$ (12/29) y en la granja 2 de $16.6 \%$ (4/24), y en heces no diarreicas se detectó una muestra positiva en cada granja. La presencia de rotavirus porcino versus la ocurrencia de diarrea, ajustada a potenciales variables confundentes (edad y procedencia) presentó un Odds Ratio de 12.6 con un IC del $95 \%$ entre 2.7 y $59.3 \%$, indicando que la presencia de rotavirus porcino representa un factor de riesgo para la ocurrencia de diarrea en lechones neonatos de granjas tecnificadas de la zona de Lima. Adicionalmente, se hallaron dos segmentos de ARN pertenecientes al genoma del Picobirnavirus porcino.
\end{abstract}

Palabras clave: diarrea, lechones, Rotavirus, electroforesis, Picobirnavirus, odds ratio

\section{Abstract}

The objective of the study was to determine the frequency of rotavirus and its association with the occurrence of diarrhea in piglets reared in two intensive pig farms in Lima valley, Peru. The presence of rotavirus was determined by the identification of the viral genome using polyacrylamide gel electrophoresis (PAGE) in diarrheic $(n=69)$ and

\footnotetext{
${ }^{1}$ Laboratorio de Microbiología y Parasitología Veterinaria, ${ }^{4}$ Laboratorio de Histología, Embriología y Patología Animal, Facultad de Medicina Veterinaria, Universidad Nacional Mayor de San Marcos, Lima

${ }^{2}$ E-mail: amanchegos@unmsm.edu.pe

${ }^{3}$ Facultad de Veterinaria y Zootecnia, Universidad Peruana Cayetano Heredia, Lima
} 
non-diarrheic $(n=73)$ fecal samples from 1 to 4 week-old piglets. The case-control epidemiological design was used to establish the association between the occurrence of diarrhea and the presence of rotavirus in feces, using a multiple logistic regression (type of feces, age, and origin). The frequency of porcine rotavirus group A in diarrheic stool samples from pig farm 1 was $41.4 \%$ (12/29) and from pig farm 2 was $16.6 \%$ (4/24), and one positive was found in non diarrheic stool from each pig farm. The presence of porcine rotavirus versus occurrence of diarrhea, adjusted to potential confusing variables (age and origin) resulted in an Odds Ratio of 12.6 with a confidence interval between 2.7 and $59.3 \%$. Additionally, two segments of RNA from the genome of porcine Picobirnavirus were found. It is concluded that the presence of porcine rotavirus represents a risk factor for the presentation of diarrhea in newborn piglets from intensive pig farms in Lima.

Key words: diarrhea, piglets, Rotavirus, electrophoresis, Picobirnavirus, odds ratio

\section{INTRODUCCIÓN}

La diarrea neonatal en lechones es una de las enfermedades de mayor importancia en países con explotaciones porcinas de crianza intensiva, debido a las considerables pérdidas económicas causadas (Ramos et al., 1998). Factores como deficiencia en el manejo, y la presencia de agentes infecciosos de origen viral, bacteriano o parasitario, desencadenan generalmente los cuadros diarreicos que pueden cursar con alta morbilidad y mortalidad (Berrios y Patricio, 1989).

Dentro de los agentes virales, los rotavirus son uno de los principales causantes de gastroenteritis en lechones en los periodos pre y post-destete, encontrándose prevalencias entre 14 y $91 \%$ (Reinhardt et $a l .$, 1986). La infección por rotavirus se caracteriza por una inflamación aguda del intestino delgado, diarrea, retardo en el crecimiento (10-15\%) y hasta $100 \%$ de mortalidad en lechones de 1 a 8 semanas de edad, aunque con mayor incidencia entre la $1^{\mathrm{a}}$ y $4^{\mathrm{a}}$ semana de edad (Bohl, 1983; Pensaert, 1984; Klug, 1993; Alfieri, 2004).

En el Perú, los problemas diarreicos en lechones son frecuentes y se ha demostrado el papel de agentes etiológicos como E. coli, Clostridium perfringens e Isospora suis, entre otros; aunque en muchos casos, estas infecciones suelen ser concomitantes o posteriores a la infección por rotavirus (Klug, 1993; Alfieri, 2004). No obstante, solo hay un estudio donde se reporta su presencia en casos de diarrea de lechones de granjas porcinas en el valle de Lima (Klug, 1993). Su identificación en los procesos diarreicos es necesaria para una mejor comprensión de la patogénesis y control de la enfermedad.

Dada la importancia del rotavirus en la patogenia de la enteritis con los consecuentes efectos en la condición general de los lechones, el objetivo del presente trabajo fue demostrar su participación en casos de síndrome diarreico agudo en dos granjas tecnificadas en la zona de Lima utilizando la electroforesis en gel de poliacrilamida (PAGE) como método diagnóstico.

\section{Materiales y Métodos}

\section{Granjas Porcinas}

El estudio se realizó en dos granjas tecnificadas de los distritos de Puente Piedra (granja 1) y Ate-Vitarte (granja 2), durante los meses de febrero a abril de 2008. La granja 1 presentaba un buen sistema de bioseguridad, poseía una población de 1200 marranas, 4000 lechones en recría y 5000 
gorrinos en engorde y acabado con 50 gorrinos por corral. La granja 2 tuvo una población de 150 marranas, 50 lechones en recría y 80 gorrinos en engorde y acabado. En ambas granjas, los lechones fueron destetados entre 21 y 24 días de edad. Durante los meses de enero a junio de 2008 se incrementaron los problemas diarreicos en un 10 a $20 \%$ en lechones de 1 a 4 semanas de edad, según informes disponibles en ambas granjas.

\section{Tamaño de Muestra}

El cálculo del tamaño muestral $(\mathrm{n}=130)$ se determinó utilizando la fórmula para estudios de caso-control no pareado (Rothman y Greenland, 1998), considerando una prevalencia de $28.3 \%$ (Barry et al., 2007), un Odds Ratio de 2.5, el nivel de confianza al $95 \%$ y un poder de la prueba de $80 \%$.

En la granja 1 se colectaron 41 muestras fecales diarreicas y 63 no diarreicas, y en la granja 2 se colectaron 28 muestras diarreicas y 10 muestras no diarreicas de lechones, en ambos casos en lechones de 1 a 4 semanas de edad y por única vez (Cuadro 1). Los lechones con y sin diarrea fueron elegidos al azar de la misma camada o de camadas distintas e indistintamente del fármaco recibido. Se colectó un mínimo de $2 \mathrm{~g}$ de heces por animal, directamente del recto. Las muestras se trasladaron al laboratorio de Virología de la Facultad de Medicina Veterinaria de la Universidad Nacional Mayor de San Marcos (FMV-UNMSM) en bolsas plásticas rotuladas, donde se almacenaron a -20 ${ }^{\circ} \mathrm{C}$ hasta su procesamiento.

\section{Extracción de ARN y Corrida Electroforética}

Se extrajo el ARN viral de las muestras de heces siguiendo el protocolo buffer de lisisfenol-cloroformo-etanol (Sulbaran y Maldonado, 2000). Una vez extraído el ARN, se procedió al montaje de la unidad de electroforesis (Bio-Rad) según las especificaciones del fabricante, y de allí se separó el
ARN genómico mediante electroforesis en gel de poliacrilamida (PAGE) al 7\%, tiñéndose con nitrato plata para identificar las 11 bandas características del genoma de rotavirus (Figs. 1 y 2).

\section{Criterio Diagnóstico}

Las muestras positivas a rotavirus fueron aquellas que mostraron las 11 bandas, toda vez que el rotavirus es el único virus que presenta 11 bandas en el PAGE al 7\% (en cada gel se coloca una muestra del control positivo con fines comparativos). Los patrones de migración de los 11 segmentos de ARN que caracteriza al rotavirus determinan el electroferotipo de las muestras positivas.

\section{Cepas de Rotavirus}

Se utilizó las cepas de rotavirus SA11 y OSU procedentes de humano y porcino, respectivamente, y una cepa de rotavirus recombinante como controles positivos.

\section{Análisis Estadístico}

Se calculó el Odds Ratio (OR) mediante el modelo de Regresión Logística múltiple incluyendo el comportamiento de las variables predictorias: condición de las heces (diarreicas y no diarreicas), procedencia (Puente Piedra y Ate Vitarte), y edad (1, 2, 3, y 4 semanas de edad), para la presentación de diagnóstico positivo a rotavirus porcino.

\section{Resultados}

En el Cuadro 2 se indica los resultados obtenidos en las muestras provenientes de ambas granjas porcinas. El 23.2\% (16/69) del total de muestras resultaron positivas a rotavirus porcino, en tanto que el $2.7 \%$ (2/ 73) del total de muestras no diarreicas fueron positivas a rotavirus (Cuadro 3). Dada la migración de los 11 segmentos RNA observado en el gel de poliacrilamida en la electroforesis, los genomas virales correspon- 


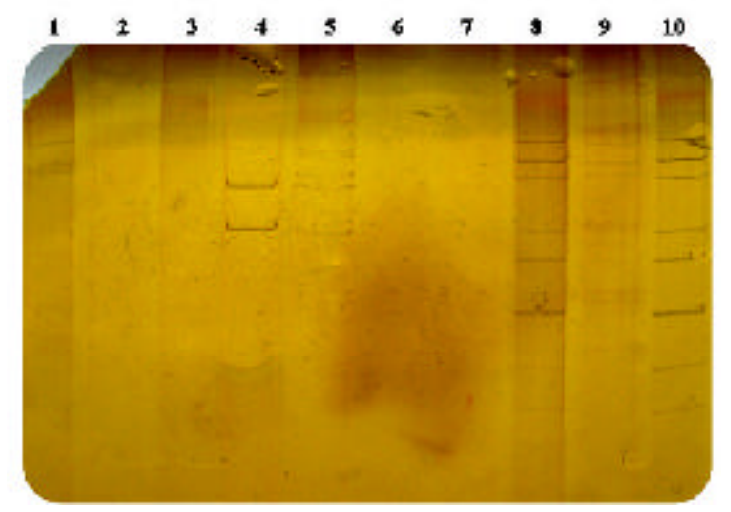

Figura 1. Gel de poliacrilamida al $7 \%$ teñido con solución de nitrato de plata. 1 al 6 , muestras de heces de lechones con diarrea; 7, control negativo; 8 y 9, muestras de heces de lechones con diarrea; 10, control de rotavirus cepa OSU. En el carril 4 se observa el genoma de un Picobirnavirus.

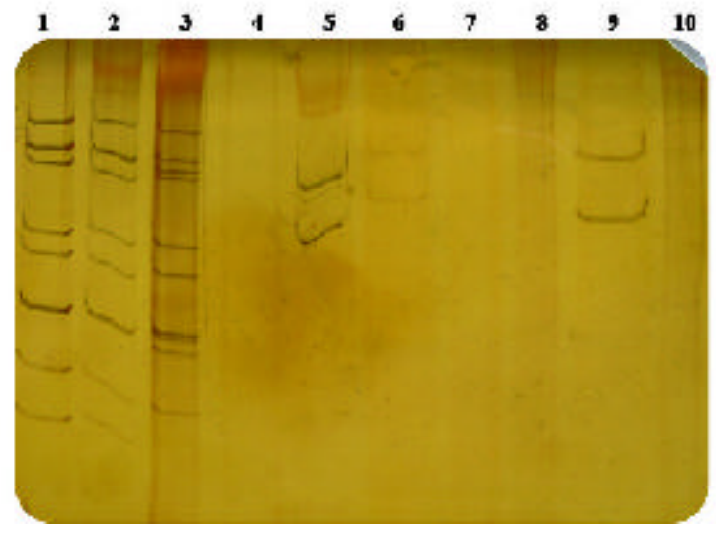

Figura 2. Gel de poliacrilamida al $7 \%$ teñido con solución de nitrato de plata. 1 y 2, muestras de heces de lechones con diarrea; 3, control positivo rotavirus recombinante (RRV); 4, control negativo; 5 y 8 , muestras de heces con diarrea; 10 , control de rotavirus cepa OSU. En el carril 6 y 9 se observa el genoma de un Picobirnavirus.

den a un electroferotipo de Rotavirus Grupo A, mostrando una característica agrupación genómica de 4-2-3-2 (Figs. 1 y 2).

Un hallazgo no previsto en el presente estudio, fue la identificación en los geles de poliacrilamida de dos segmentos de ARN pertenecientes al genoma del Picobirnavirus porcino. Esto fue observado con mayor claridad en muestras negativas a rotavirus (Fig. 1 y 2). Estos genomas se presentaron con una frecuencia de $5.6 \%$ (8/142), ocurriendo solamente en lechones de 3 y 4 semanas de edad. El rol de este agente en la salud de los gorrinos requiere ser estudiado. 


\section{Discusión}

El rotavirus porcino fue detectado en el $41.4 \%(12 / 29)$ y $16.6 \%(4 / 24)$ de muestras diarreicas de lechones de las granjas 1 y 2 , respectivamente, mientras que solo dos lechones fueron positivos en el grupo de lechones sin diarrea de ambas granjas (Cuadro 2). Estos resultados indican que el rotavirus porcino fue uno de los agentes productores de diarrea en los lechones de las granjas porcinas muestreadas. El presente resultado es similar a las informaciones provenientes de otros países con gran desarrollo de la industria porcina, en donde el rotavirus porcino alcanza prevalencias entre $14 \%$ a más de $90 \%$ (Reinhardt et al., 1986; Barry et al., 2007). En la granja 2, la ocurrencia de diarrea en lechones fue más de $20 \%$ durante los meses del muestreo, pero solo $13.2 \%$ (5/38) de las muestras fueron positivas al rotavirus indicando que, además del rotavirus, pudo existir otras causas o factores que ocasionaron el incremento de diarreas.

La frecuencia de $23.2 \%$ de rotavirus porcino en heces de lechones con diarrea versus a $2.7 \%$ en lechones sin diarrea indica un alto grado de asociación entre la presencia de rotavirus porcino y la diarrea en lechones, en las dos granjas tecnificadas de la zona de Lima (Cuadro 3). En algunos países de América Latina, donde la industria porcina ha alcanzado un gran desarrollo, como Chile y Brasil, el rotavirus es considerado como uno de los agentes infecciosos más importantes causante de diarreas en lechones (Rivas, 1983; Reinhardt et al., 1986; Alfieri et al., 2006; Barry et al., 2007).

El $2.7 \%(2 / 73)$ de las muestras sin diarreas resultaron positivas a rotavirus, sugiriendo que la infección por rotavirus en ambas granjas estuvo difundiéndose entre los lechones a pesar de las medidas de bioseguridad, pero también se debe considerar que el rotavirus puede infectar lechones en forma subclínica, aunque en una menor frecuencia. Polanco et al. (2004) reportaron una prevalencia de rotavirus del $3.3 \%$ en lechones sin diarrea. La presencia de animales asintomáticos es importante en la epidemiología del rotavirus, ya que estos lechones son fuente del virus para los animales susceptibles, como los lechones recién nacidos (Alfieri, 2004).

Los resultados indican, además, que el rotavirus representa un factor de riesgo para la presentación de diarrea en lechones neonatos en granjas tecnificadas de la zona de Lima (Cuadro 4). Asimismo, los datos muestran un potencial riesgo de infección para otros animales y, posiblemente, para las personas que están en contacto con estos animales en las granjas, ya que este virus ha sido reconocido como una posible entidad zoonótica (Acha y Syfres, 2003).

La técnica de electroforesis (PAGE) en gel de poliacrilamida fue usada en el presente estudio por ser una de las técnicas de diagnóstico molecular de mayor confiabilidad, con una sensibilidad de $95 \%$ y una especificidad de 100\% (Sulbaran y Maldonado, 2000; Nusetti et al., 2001). Además, es un método económico y práctico en comparación de otros métodos no asequibles en el país por su elevado costo y dificultad en el manejo de numerosas muestras para el diagnóstico, como es el caso de la microscopía electrónica (Iwona et al., 1996; Nusetti et al., 2001). Asimismo, los inmunoensayos enzimáticos comerciales (ELISA), si bien tienen alta sensibilidad (95-100\%) que permiten diferenciar los serotipos del grupo "A", tienen baja especificidad (75\%), no permitiendo diferenciar los grupos de los rotavirus (Iwona et al., 1996; Sulbaran y Maldonado, 2000; Nusetti et al., 2001).

El hallazgo de Picobirnavirus (PBV) porcino en los geles SDS PAGE es coincidente con hallazgos accidentales del genoma de PBV en Brasil, Venezuela y Argentina (Gatti et al., 1989; Ludert, 1994; Giordano et al., 1998). El PBV es un virus relativamente nuevo, ya que se descubrió en 1988, y hasta la fecha no se conoce bien su rol patógeno 
Cuadro 1. Número de lechones con y sin diarrea, con muestras de heces colectadas, de dos granjas porcinas de la zona de Lima

\begin{tabular}{ccccccc}
\hline \multirow{2}{*}{$\begin{array}{c}\text { Edad } \\
\text { (semanas) }\end{array}$} & \multicolumn{3}{c}{ Granja 1 } & \multicolumn{3}{c}{ Granja 2 } \\
\cline { 2 - 7 } & Con diarrea & Sin diarrea & Total & Con diarrea & Sin diarrea & Total \\
\hline 1 & 2 & 12 & 14 & 4 & 3 & 7 \\
2 & 7 & 20 & 27 & 3 & 5 & 8 \\
3 & 20 & 17 & 37 & 15 & 1 & 16 \\
4 & 12 & 14 & 26 & 6 & 1 & 7 \\
\hline Total & 41 & 63 & 104 & 28 & 10 & 38 \\
\hline
\end{tabular}

Cuadro 2. Frecuencia de lechones positivos a rotavirus porcino en dos granjas porcinas de la zona de Lima

\begin{tabular}{cccccc}
\hline \multirow{2}{*}{$\begin{array}{c}\text { Granja } \\
\text { porcina }\end{array}$} & $\begin{array}{c}\text { Edad } \\
\text { (semanas) }\end{array}$ & \multicolumn{2}{c}{ Lechones con diarrea } & \multicolumn{2}{c}{ Lechones sin diarrea } \\
\cline { 2 - 6 } & 1 & 1 & 1 & 12 & 0 \\
\hline \multirow{3}{*}{ Granja 1} & 2 & 4 & 3 & 20 & 0 \\
& 3 & 15 & 5 & 17 & 0 \\
& 4 & 9 & 3 & 13 & 1 \\
\cline { 2 - 6 } & Total & 29 & $12(41.4 \%)$ & 9 & $1(1.6 \%)$ \\
\hline \multirow{3}{*}{ Granja 2 } & 1 & 4 & 0 & 3 & 0 \\
& 2 & 3 & 0 & 5 & 0 \\
& 3 & 12 & 3 & 1 & 0 \\
& 4 & 5 & 1 & 0 & 1 \\
\hline & Total & 24 & $4(16.6 \%)$ & 62 & $1(11.1 \%)$ \\
\hline
\end{tabular}

en animales y el humano, pero se sabe que está presente en todos los vertebrados (Pereira et al., 1988; Ludert, 1994). Se le encuentra mayormente en cuadros de diarrea en animales y humanos con inmunodepresión, por lo que es considerado como el segundo agente patógeno en importancia causante de diarrea en humanos con VIH (Liste et al.,
2000). En el presente estudio, se encontró la misma frecuencia de PBV en lechones con $(5.8 \%)$ y sin diarrea (5.5\%), indicando que el virus no sería el causante de la diarrea; sin embargo, hay otros reportes que sugieren que este virus podría ser causante de diarreas en porcinos (Gatti et al., 1989). 
Cuadro 3. Distribución de muestras positivas a rotavirus porcino por el método de electroforesis en gel de poliacrilamida (PAGE), según la presencia o ausencia de diarrea, localidad de procedencia y edad de los lechones

\begin{tabular}{lcccc}
\hline \multirow{2}{*}{ Variable } & $\begin{array}{c}\text { Estratos de la } \\
\text { Variable }\end{array}$ & $\begin{array}{c}\text { Total de } \\
\text { muestras }\end{array}$ & \multicolumn{2}{c}{ Muestras positivas } \\
\cline { 5 - 6 } & Deces & & $\mathrm{n}$ & $\%$ \\
& Diarreica & 69 & 16 & $23.2^{\mathrm{a}}$ \\
& No diarreica & 73 & 2 & $2.7^{\mathrm{b}}$ \\
Procedencia & & & & \\
& Puente Piedra & 104 & 13 & $12.5^{\mathrm{a}}$ \\
& Ate Vitarte & 38 & 5 & $13.2^{\mathrm{a}}$ \\
Edad & & & & \\
(semanas) & 1 & 21 & 1 & $4.8^{\mathrm{a}}$ \\
& 2 & 35 & 3 & $8.6^{\mathrm{a}}$ \\
& 3 & 53 & 8 & $15.1^{\mathrm{a}}$ \\
& 4 & 33 & 6 & $18.2^{\mathrm{a}}$ \\
\hline Total & & 142 & 18 & 12.7 \\
\hline
\end{tabular}

${ }^{a, b}$ Superíndices diferentes dentro de una variable indican diferencia estadística $(p>0.05)$

\section{Conclusiones}

? La presencia de rotavirus porcino representa un factor de riesgo para la presentación de diarrea en lechones neonatos de granjas tecnificadas de la zona de Lima.

? El $2.7 \%$ de lechones asintomáticos resultaron positivos a rotavirus.

? Se reporta la presencia de picobirnavirus porcino en lechones.

\section{Agradeciemientos}

Los autores agradecen a Mariluz Arainga y Kim Lam Chiok por la asesoría prestada en el desarrollo de la técnica de PAGE.

\section{Literatura Citada}

1. Acha P, Syfres B. 2003. Zoonosis y enfermedades transmisibles comunes al hombre y a los animales. $3^{\text {a }}$ ed. Washington OPS. 398 p.

2. Alfieri AA. 2004. Epidemiología de la Rotavirosis Porcina. Av Tecnol Porc 2(78): $16-24$

3. Alfieri AA, Parazzi ME, Takiuchi E, Médici KC, Alfieri AF. 2006. Frequency of group A rotavirus in diarrhoeic calves in Brazilian cattle herds, 1998-2002. Trop Anim Health Prod 38: 521-526.

4. Barry AF, Linares RC, Alfieri AF, Feronato C, Grieder W, Alfieri AA. 2007. Frequency rate of group "A" Rotavirus in diarrheic suckling piglets from unvaccinated Brazilian pig herds. 
In: Anais do XIII Congresso Brasileiro de Veterinários Especialistas em Suínos. Florianópolis: ABRAVES.

5. Berrios E, Patricio P. 1989. Presencia de Rotavirus en cerdos lactantes con sindrome diarreico. Avances Med Vet 4(2): 215-228.

6. Bohl FH. 1983. Viral diarrhea in piglets. In: Annual Meeting AASP. Cincinnati, Ohio: American Association of Swine Practitioners.

7. Gatti MS, de Castro AF, Ferraz MM, Fialho AM, Pereira HG. 1989. Viruses with bisegmented double-stranded RNA in pig faeces. Res Vet Sci 47: 397-398.

8. Giordano MO, Martinez LC, Rinaldi D, Guinard S, Nasaretto E, Casero R, Yacci MR, et al. 1998. Detection of Picobirnavirus in HIV-infected patients with diarrehea in Argentina. J Acq Immun Def Synd 18: 380-383.

9. Iwona M-D, Winiarczyk S, Gradzki Z, Pejsak Z. 1996. Evalaution of different methods (ELISA, IF, EM, PAGE) for the diagnosis of rotavirus infection in piglets. Comp Immunol Microbiol 19: 219-232.

10. Klug H. 1993. RNA-PAGE en la detección de enteritis porcina por rotavirus. Tesis de Médico Veterinario. Lima: Facultad de Medicina Veterinaria, Univ Nacional Mayor de San Marcos. $18 \mathrm{p}$.

11. Liste MB, Natera I, Suarez JA, Pujol FH, Liprandi F, Ludert JE. 2000. Enteric virus infections and diarrhea in healthy and human immunodeficiency virus-infected children. J Clin Microbiol 38: 2873-2877.

12. Ludert JE. 1994. El viaje inverso: El descubrimiento de los Picobirnavirus. Interciencia 19(3): 127-129. [Internet].
Disponible en: http//www. intercencia.org.ve

13. Nusetti S, Maldonado A, Bastardo J. 2001. Comparación de tres métodos diagnósticos para la detección de Rotavirus en heces de niños diarreicos. Saber, Venezuela 13(1): 38-41.

14. Pensaert MB. 1984. Viral gastroenteritis in suckling pigs. Rev Sci Tech Off Int Epiz 3: 809-818.

15. Pereira HG, Flewet TH, Candeias JAN, Barth OM. 1988. A virus with a bisegmented double-stranded RNA genome in rat (Oryzomys nigripes) intestines. J Gen Virol 69: 2749-2754.

16. Polanco G, González M, Manzano L. 2004. Rotavirus en animales asintomáticos: Detección y clasificación antigénica, Arch Med Vet 36(1): 65-70.

17. Ramos AP, Stefanelli C, Linhares RE. 1998. The infectivity of pig rotavirus in stools. Braz J Vet Res Anim Sci 35(2): 84-87.

18. Reinhardt G, Riedmann S, Polette M, Aguilar M. 1986. Diarrea neonatal: infección por rotavirus en bovinos y porcinos. Arch Med Vet 18: 23-27.

19. Rivas C. 1983. Detección y caracterización de rotavirus en cerdos lactantes en la Región Metropolitana de Santiago de Chile. Tesis de Médico Veterinario. Santiago: Universidad de Chile. 45 p.

20. Rothman K, Greenland S. 1998. Modern epidemiology. $2^{\text {nd }}$ ed. Philadelphia: Lippincott Williams \& Wilking. $738 \mathrm{p}$.

21. Sulbaran MZ, Maldonado AJ. 2000. Método rápido de diagnóstico de rotavirus en heces. Saber, Venezuela. 12(2): 29-36. 